\title{
Postoperative outcomes for Indigenous Peoples in Canada: a systematic review
}

\author{
Jason A. McVicar MD, Alana Poon MD, Nadine R. Caron MD MPH, M. Dylan Bould MBChB Med, \\ Jason W. Nickerson RRT PhD, Nora Ahmad MD, Donna May Kimmaliardjuk MD, Chelsey Sheffield MD, \\ Caitlin Champion MD MSc, Daniel I. Mclsaac MD MPH
}

Cite as: CMAJ 2021 May 17;193:E713-22. doi: 10.1503/cmaj.191682

\section{ABSTRACT \\ Background: Substantial health inequities exist for Indigenous Peoples in Canada. The remote and distributed population of Canada presents unique challenges for access to and use of surgery. To date, the surgical outcome data for Indigenous Peo- ples in Canada have not been synthesized.}

Methods: We searched 4 databases to identify studies comparing surgical outcomes and utilization rates of adults of First Nations, Inuit or Métis identity with non-Indigenous people in Canada. Independent reviewers completed all stages in duplicate. Our primary outcome was mortality; secondary outcomes included utilization rates of surgical procedures, complications and hospital length of stay. We performed meta- analysis of the primary outcome using random effects models. We assessed risk of bias using the ROBINS-I tool.

Results: Twenty-eight studies were reviewed involving 1976258 participants (10.2\% Indigenous). No studies specifically addressed Inuit or Métis populations. Four studies, including 7 cohorts, contributed adjusted mortality data for 7135 participants (5.2\% Indigenous); Indigenous Peoples had a 30\% higher rate of death after surgery than nonIndigenous patients (pooled hazard ratio $1.30,95 \%$ Cl 1.09-1.54; $P=81 \%$ ). Complications were also higher for Indigenous Peoples, including infectious complications (adjusted OR 1.63, 95\% Cl 1.13-2.34) and pneumonia (OR 2.24, 95\% Cl 1.58-
3.19). Rates of various surgical procedures were lower, including rates of renal transplant, joint replacement, cardiac surgery and cesarean delivery.

Interpretation: The currently available data on postoperative outcomes and surgery utilization rates for Indigenous Peoples in Canada are limited and of poor quality. Available data suggest that Indigenous Peoples have higher rates of death and adverse events after surgery, while also encountering barriers accessing surgical procedures. These findings suggest a need for substantial re-evaluation of surgical care for Indigenous Peoples in Canada to ensure equitable access and to improve outcomes. Protocol registration: PROSPERO- CRD42018098757

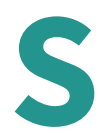

afe, timely and affordable access to surgical care is essential to overall population health, as conditions amenable to surgical intervention account for one-third of the global burden of disease. ${ }^{1,2}$ Surgery is responsible for $65 \%$ of cancer cure and control, it is key to trauma management, and access to cesarean delivery reduces neonatal deaths by up to $70 \% .{ }^{1}$ The magnitude and ubiquity of surgical conditions makes tracking their prevalence and treatment within local and national monitoring systems essential to fully capture the health and welfare of populations in Canada, including Indigenous Peoples.

About 1.67 million people in Canada are Indigenous, representing $4.9 \%$ of the total population (58\% First Nations, 4\% Inuit, $35 \%$ Métis). ${ }^{3}$ Health inequities exist for the Indigenous population; life expectancy at birth is 5-11 years shorter than for nonIndigenous Peoples ${ }^{4,5}$ and higher rates of communicable and noncommunicable diseases, unintentional injury and suicide are well documented. ${ }^{4,6-14}$ These health inequities are direct impacts of the social determinants of health, which are in turn effects of colonialism and government policies, including the Indian residential school system. ${ }^{8,11}$ People living in remote regions have less access to publicly funded health care than other people in Canada, with worse outcomes. ${ }^{15}$

Given the substantial impact of surgical disease on population health and the recognized disparities in health care access for Indigenous Peoples in Canada, understanding access to surgical services and subsequent outcomes is a key step to addressing health inequities. To date, limited research has been conducted on surgical and postoperative care involving Indigenous Peoples in Canada and the available literature has not been synthesized. Our objective was to systematically review studies comparing postoperative outcomes between Indigenous and nonIndigenous Peoples in Canada. 


\section{Methods}

Following protocol registration with the International Prospective Register of Systematic Reviews (CRD42018098757), we conducted a systematic review in accordance with the Cochrane Collaboration and Meta-analysis Of Observational Studies in Epidemiology (MOOSE) guidelines. ${ }^{16}$ We report our findings according to the Preferred Reporting Items for Systematic Reviews and Meta-Analysis (PRISMA) statement. ${ }^{17}$

\section{Search strategy}

We constructed a comprehensive and peer-reviewed ${ }^{18}$ search strategy, in collaboration with an information specialist, using terms related to surgery and identification of Indigenous Peoples in Canada. We applied our search strategy to MEDLINE, Embase, Cochrane and the Cumulative Index to Nursing \& Allied Health Literature without language restriction, from inception to Apr. 12, 2019 (Appendix 1, available at www.cmaj.ca/lookup/ doi/10.1503/cmaj.191682/tab-related-content). We reviewed the reference lists of included articles. We included all languages; however, we did not search the grey literature because of variability of quality, high risk of bias and limited ability to verify methods. We contacted when clarification was required.

\section{Outcomes}

Our primary outcome was all-cause postoperative mortality; we did not limit the window of outcome ascertainment. Secondary outcomes included complications, length of stay, wait times and rates of surgery, resource use, satisfaction and quality of life.

\section{Inclusion and exclusion criteria}

We included studies if they addressed adults having surgery or cesarean deliveries in Canada, identified Indigenous Peoples by any method (e.g., an explicit measurement technique or through self-identification) and provided quantitative data making a comparison between a group with Indigenous identity and one without Indigenous identity or the general population with respect to primary or secondary outcomes. We excluded studies that addressed non-Canadian or non-Indigenous Peoples and case reports or series.

\section{Study selection and data extraction}

Two independent reviewers (A.P. and N.A.) performed all stages of study selection in duplicate using Covidence systematic review software (Veritas Health Innovation). Reviewers first screened titles and abstracts. We specified that both reviewers must agree to exclude a study; any disagreements or uncertainties were advanced to full text review where disagreements were resolved by consensus (A.P., N.A., J.M., D.M.). Two independent reviewers (A.P. and N.A.) extracted data of studies included after full text review using a form specifically designed and piloted for this study. We extracted publication details, study population characteristics, design and outcomes data (number, proportion, central measures of tendency and variance), as well as rates of outcomes and unadjusted and adjusted effect sizes (i.e., odds ratios [ORs], risk ratios [RRs] and hazard ratios [HRs]). We also extracted data on key confounders including age, comorbidities, rural or urban residence and income. For obstetric studies, we extracted data on maternal outcomes only.

\section{Data analysis}

We summarized study characteristics descriptively. Primary outcome data, adjusted for prespecified confounders (procedure, age, and comorbidity), were meta-analyzed using models weighted for random effects inverse variance to account for pooling across heterogenous surgical procedures (Comprehensive Meta-Analysis software, Biostat). We assessed heterogeneity using the $I^{2}$ statistic. We performed a narrative synthesis of other outcome data.

Two independent reviewers (D.M. and J.M.) assessed risk of bias using the Risk Of Bias In Non-Randomized Studies of Interventions (ROBINS-I) tool. ${ }^{19}$ We particularly focused on study definitions of exposure status, as described in Appendix 2, available at www.cmaj. ca/lookup/doi/10.1503/cmaj.191682/tab-related-content.

\section{Results}

We identified 707 titles and abstracts, reviewed 118 full-text articles and included 28 studies (Figure 1, Table 1) published in English from 1989 to 2016. Surgical specialties included urologic $(n=8,29 \%),{ }^{25,34,40-43,45,46}$ obstetric $(n=5,18 \%),{ }^{29,33,35,38}$ orthopedic $(n=3,11 \%),{ }^{21,28,30,32}$ general $(n=3,11 \%),{ }^{24,27,47}$ cardiac $(n=3$, $11 \%),{ }^{22,39,44}$ ophthalmologic $(n=1,4 \%)$, plastic $(n=1,4 \%)$ and vascular $(n=1,4 \%)$ surgery. ${ }^{23,26,37}$ Three studies (11\%) included a mix of surgical specialties. ${ }^{31,36}$ All studies used observational designs (26 retrospective cohorts, 1 case-control, 1 crosssectional). Of 1976258 participants from all included studies, 202056 (10.2\%) identified as Indigenous. Studies used a variety of methods to identify Indigenous identity (Appendix 3, available at www.cmaj.ca/lookup/doi/10.1503/cmaj.191682/tab -related-content).

\section{Outcomes}

Eight studies reported mortality rates (Table 2), ${ }^{22,23,26-28,39,43,47}$ including 4 studies that reported crude mortality data. ${ }^{22,23,26,47}$ Overall, $47(16.1 \%)$ of 292 people of Indigenous identity died, compared with 1209 (21.4\%) of 5647 people of non-Indigenous identity; however, inadequate description of unadjusted data precluded meta-analysis. Meta-analysis of adjusted data estimated a pooled HR of 1.30 (95\% confidence interval $[\mathrm{Cl}] 1.09-$ $1.54, P^{2}=81 \%$ ), where an HR $>1$ indicates a higher risk of death for Indigenous Peoples (Figure 2). One study reported increased mortality for the Indigenous cohort using adjusted ORs (OR 1.15, $95 \% \mathrm{Cl} 0.63-2.08$ ), but the trend was not statistically significant. ${ }^{39}$ We explored possible sources of heterogeneity in our pooled effect by surgery type (orthopedic, transplant and cardiovascu(ar). The percentage of variation attributed to heterogeneity across studies was $0 \%$ for orthopedic and cardiovascular surgeries and $89 \%$ for transplant surgeries. When we pooled cardiovascular and orthopedic surgeries, the pooled HR estimate was 1.33 $\left(95 \% \mathrm{Cl} 1.16-1.54, I^{2}=37 \%\right)$; pooled transplant studies had an HR of $1.41\left(95 \% \mathrm{Cl} 0.81-2.46, I^{2}=89 \%\right)$. A sensitivity analysis explored 


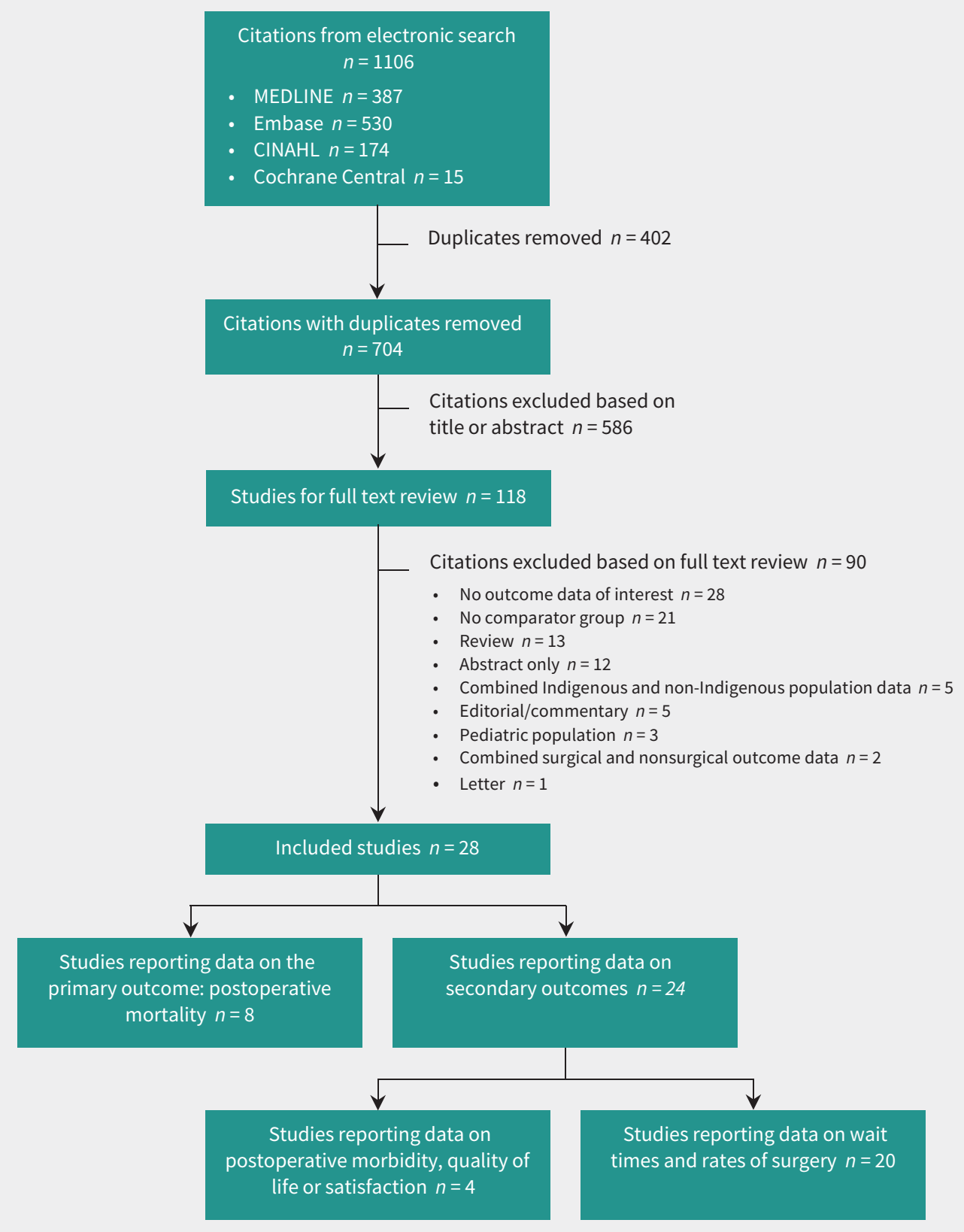

Figure 1: Flow diagram of study selection. CINAHL = Cumulative Index to Nursing \& Allied Health Literature.

the role of follow-up time as an effect modifier using metaregression; we found no evidence of effect modification $(p=0.317)$.

Twenty-four studies reported on secondary outcomes. The wide range of secondary outcomes is described in Appendix 4, available at www.cmaj.ca/lookup/doi/10.1503/cmaj.191682/tab -related-content. Complications were routinely reported and were higher for Indigenous Peoples. After cardiac surgery, we found higher adjusted rates of infectious complications among First Nations Peoples (infection-adjusted OR 1.63, 95\% Cl 1.132.34; pneumonia OR 2.24, $95 \% \mathrm{Cl}$ 1.58-3.19); a composite of major cardiac and renal events did not differ. ${ }^{39}$ Living kidney donors who were First Nations or Métis had higher rates of longterm complications, such as new hypertension (adjusted OR 6.3, $95 \% \mathrm{Cl} 1.8-22.1$ ), and diabetes (19.4\% v. $1.6 \%, p=0.005) .{ }^{40}$ Kidney transplant recipients (HR 1.53, 95\% Cl 1.20-1.95) were more likely to experience graft failure. ${ }^{25}$ After cholecystectomy, First Nations identity was associated with an adjusted 1.5 -fold increase in the odds of hospital readmission $(95 \% \mathrm{Cl} 1.17-1.81) .^{24}$ We assessed quality of life after diabetic limb amputation and noted no difference between patients of First Nations or Métis identity and the rest of the study participants (decreased in both groups). ${ }^{32}$ 


\begin{tabular}{|c|c|c|c|c|c|c|c|c|}
\hline Study & Study design & $\begin{array}{l}\text { Surgical } \\
\text { specialty }\end{array}$ & $\begin{array}{l}\text { Sample } \\
\text { size }\end{array}$ & $\begin{array}{l}\text { No. } \\
\text { Indigenous }\end{array}$ & $\begin{array}{c}\text { No. } \\
\text { non- } \\
\text { Indigenous }\end{array}$ & $\begin{array}{l}\text { Indigenous } \\
\text { population }\end{array}$ & $\begin{array}{l}\text { Identification of } \\
\text { Indigenous } \\
\text { population }\end{array}$ & Outcome of interest \\
\hline $\begin{array}{l}\text { Aljohani et } \\
\text { al. }^{20}\end{array}$ & $\begin{array}{l}\text { Retrospective } \\
\text { cohort study }\end{array}$ & Obstetric & 165969 & 20414 & 145555 & $\begin{array}{l}\text { First } \\
\text { Nations }\end{array}$ & $\begin{array}{l}\text { First Nations } \\
\text { status }\end{array}$ & $\begin{array}{l}\text { Rates of cesarean } \\
\text { delivery }\end{array}$ \\
\hline $\begin{array}{l}\text { Barnabe et } \\
\text { al. }^{21}\end{array}$ & $\begin{array}{l}\text { Retrospective } \\
\text { cohort study }\end{array}$ & Orthopedic & 300673 & 10745 & 289928 & $\begin{array}{l}\text { First } \\
\text { Nations, } \\
\text { Inuit and } \\
\text { Métis }\end{array}$ & $\begin{array}{l}\text { Payment through } \\
\text { the First Nations } \\
\text { and Inuit Health } \\
\text { Branch (Health } \\
\text { Canada) }\end{array}$ & $\begin{array}{l}\text { Rates of hip or knee } \\
\text { arthroplasty } \\
\text { for osteoarthritis }\end{array}$ \\
\hline Bresee et al. ${ }^{22}$ & $\begin{array}{l}\text { Retrospective } \\
\text { cohort study }\end{array}$ & Cardiac & 22171 & 489 & 21682 & $\begin{array}{l}\text { First } \\
\text { Nations }\end{array}$ & $\begin{array}{l}\text { First Nations } \\
\text { status }\end{array}$ & $\begin{array}{l}\text { Rates of coronary } \\
\text { revascularization after } \\
\text { acute myocardial } \\
\text { infarction; death after } \\
\text { coronary } \\
\text { revascularization for } \\
\text { acute myocardial } \\
\text { infarction }\end{array}$ \\
\hline $\begin{array}{l}\text { Callegari et } \\
\text { al. }^{23}\end{array}$ & $\begin{array}{l}\text { Retrospective } \\
\text { cohort study }\end{array}$ & Plastic & 1598 & 125 & 1473 & $\begin{array}{l}\text { First } \\
\text { Nations and } \\
\text { Métis }\end{array}$ & Unknown & $\begin{array}{l}\text { Rates of surgical } \\
\text { treatment for burns }\end{array}$ \\
\hline $\begin{array}{l}\text { Cohen et al. } \\
1989^{24}\end{array}$ & $\begin{array}{l}\text { Retrospective } \\
\text { cohort study }\end{array}$ & General & 37383 & 1327 & 36056 & $\begin{array}{l}\text { First } \\
\text { Nations }\end{array}$ & $\begin{array}{l}\text { First Nations } \\
\text { status }\end{array}$ & $\begin{array}{l}\text { Readmission rate after } \\
\text { cholecystectomy }\end{array}$ \\
\hline Dyck $^{25}$ & $\begin{array}{l}\text { Retrospective } \\
\text { cohort study }\end{array}$ & Urologic & 645 & 89 & 556 & $\begin{array}{l}\text { Not } \\
\text { specified }\end{array}$ & $\begin{array}{l}\text { First Nations } \\
\text { status }\end{array}$ & Rates of renal transplant \\
\hline Goulet et al. ${ }^{26}$ & $\begin{array}{l}\text { Retrospective } \\
\text { cohort study }\end{array}$ & Vascular & 678 & 84 & 594 & $\begin{array}{l}\text { First } \\
\text { Nations, } \\
\text { Inuit, Métis }\end{array}$ & $\begin{array}{l}\text { First Nations } \\
\text { status or ethnicity } \\
\text { referenced in } \\
\text { medical record }\end{array}$ & $\begin{array}{l}\text { Death after } \\
\text { revascularization for } \\
\text { peripheral vascular } \\
\text { disease }\end{array}$ \\
\hline Hong et al..$^{27}$ & $\begin{array}{l}\text { Retrospective } \\
\text { cohort study }\end{array}$ & General & 1164 & Unknown & Unknown & $\begin{array}{l}\text { Not } \\
\text { specified }\end{array}$ & $\begin{array}{l}\text { Canadian Organ } \\
\text { Replacement } \\
\text { Register }\end{array}$ & $\begin{array}{l}\text { Death after liver } \\
\text { transplant }\end{array}$ \\
\hline Leslie et al. ${ }^{28}$ & $\begin{array}{l}\text { Retrospective } \\
\text { case control }\end{array}$ & Orthopedic & 104292 & 1069 & 103223 & $\begin{array}{l}\text { First } \\
\text { Nations }\end{array}$ & $\begin{array}{l}\text { First Nations } \\
\text { status }\end{array}$ & $\begin{array}{l}\text { Death after } \\
\text { surgery for nontraumatic } \\
\text { hip, wrist or spine fracture }\end{array}$ \\
\hline Liu et al. ${ }^{29}$ & $\begin{array}{l}\text { Retrospective } \\
\text { cohort study }\end{array}$ & Obstetric & 29216 & 253 & 28963 & $\begin{array}{l}\text { First } \\
\text { Nations }\end{array}$ & $\begin{array}{l}\text { On reserve First } \\
\text { Nations residence }\end{array}$ & $\begin{array}{l}\text { Rates of cesarean } \\
\text { delivery }\end{array}$ \\
\hline $\begin{array}{l}\text { Martens et } \\
\text { al. }^{30}\end{array}$ & $\begin{array}{l}\text { Cross-sectional } \\
\text { study }\end{array}$ & Orthopedic & Unknown & 116071 & Unknown & $\begin{array}{l}\text { First } \\
\text { Nations }\end{array}$ & $\begin{array}{l}\text { First Nations } \\
\text { status }\end{array}$ & $\begin{array}{l}\text { Rates of amputation in } \\
\text { patients with diabetes }\end{array}$ \\
\hline $\begin{array}{l}\text { Mclntyre et } \\
\text { al. }^{31}\end{array}$ & $\begin{array}{l}\text { Retrospective } \\
\text { cohort study }\end{array}$ & $\begin{array}{l}\text { Orthopedic, } \\
\text { vascular }\end{array}$ & 127 & 64 & 63 & $\begin{array}{l}\text { First } \\
\text { Nations, } \\
\text { Métis }\end{array}$ & Self-identified & $\begin{array}{l}\text { Rates of amputation or } \\
\text { vascular bypass in } \\
\text { patients on dialysis }\end{array}$ \\
\hline Meatherall ${ }^{32}$ & $\begin{array}{l}\text { Retrospective } \\
\text { cohort study }\end{array}$ & Orthopedic & 44 & 21 & 23 & $\begin{array}{l}\text { First } \\
\text { Nations, } \\
\text { Métis }\end{array}$ & Unknown & $\begin{array}{l}\text { Disability and quality } \\
\text { of life after lower } \\
\text { limb amputation }\end{array}$ \\
\hline Oster et al. ${ }^{33}$ & $\begin{array}{l}\text { Retrospective } \\
\text { cohort study }\end{array}$ & Obstetric & 427058 & 28306 & 398752 & $\begin{array}{l}\text { First } \\
\text { Nations }\end{array}$ & $\begin{array}{l}\text { First Nations } \\
\text { status }\end{array}$ & $\begin{array}{l}\text { Rates of cesarean } \\
\text { delivery }\end{array}$ \\
\hline $\begin{array}{l}\text { Promislow et } \\
\text { al. }^{34}\end{array}$ & $\begin{array}{l}\text { Retrospective } \\
\text { cohort study }\end{array}$ & Urologic & 30688 & 2361 & 28327 & $\begin{array}{l}\text { Not } \\
\text { specified }\end{array}$ & $\begin{array}{l}\text { Canadian Organ } \\
\text { Replacement } \\
\text { Register }\end{array}$ & $\begin{array}{l}\text { Rates of renal } \\
\text { transplantation }\end{array}$ \\
\hline Riddell et al..$^{35}$ & $\begin{array}{l}\text { Retrospective } \\
\text { cohort study }\end{array}$ & Obstetric & 215993 & 9152 & 206841 & $\begin{array}{l}\text { First } \\
\text { Nations }\end{array}$ & $\begin{array}{l}\text { First Nations } \\
\text { status }\end{array}$ & $\begin{array}{l}\text { Rates of cesarean } \\
\text { delivery }\end{array}$ \\
\hline Rose et al. ${ }^{36}$ & $\begin{array}{l}\text { Retrospective } \\
\text { cohort study }\end{array}$ & $\begin{array}{l}\text { Orthopedic, } \\
\text { vascular }\end{array}$ & 325 & 224 & 101 & $\begin{array}{l}\text { First } \\
\text { Nations, } \\
\text { Métis }\end{array}$ & Unknown & $\begin{array}{l}\text { Rates of amputation in } \\
\text { patients with diabetes }\end{array}$ \\
\hline Roy et al. ${ }^{37}$ & $\begin{array}{l}\text { Retrospective } \\
\text { cohort study }\end{array}$ & Ophthalmologic & 88 & 43 & 45 & $\begin{array}{l}\text { First } \\
\text { Nations }\end{array}$ & Unknown & $\begin{array}{l}\text { Rates of surgical } \\
\text { treatment for uveitis }\end{array}$ \\
\hline Shen et al. ${ }^{38}$ & $\begin{array}{l}\text { Retrospective } \\
\text { cohort study }\end{array}$ & Obstetric & 214028 & Unknown & Unknown & $\begin{array}{l}\text { First } \\
\text { Nations }\end{array}$ & $\begin{array}{l}\text { First Nations } \\
\text { status }\end{array}$ & Rates of cesarean delivery \\
\hline
\end{tabular}




\begin{tabular}{|c|c|c|c|c|c|c|c|c|}
\hline Study & Study design & $\begin{array}{l}\text { Surgical } \\
\text { specialty }\end{array}$ & $\begin{array}{l}\text { Sample } \\
\text { size }\end{array}$ & $\begin{array}{l}\text { No. } \\
\text { Indigenous }\end{array}$ & $\begin{array}{c}\text { No. } \\
\text { non- } \\
\text { Indigenous }\end{array}$ & $\begin{array}{l}\text { Indigenous } \\
\text { population }\end{array}$ & $\begin{array}{l}\text { Identification of } \\
\text { Indigenous } \\
\text { population }\end{array}$ & Outcome of interest \\
\hline Sood et al. ${ }^{39}$ & $\begin{array}{l}\text { Retrospective } \\
\text { cohort study }\end{array}$ & Cardiac & 12170 & 574 & 11596 & $\begin{array}{l}\text { First } \\
\text { Nations, } \\
\text { Inuit, Métis }\end{array}$ & Self-identified & $\begin{array}{l}\text { Rates of cardiac surgery; } \\
\text { Morbidity after cardiac } \\
\text { surgery; death after } \\
\text { cardiac surgery }\end{array}$ \\
\hline $\begin{array}{l}\text { Storsely et } \\
\text { al. }{ }^{40}\end{array}$ & $\begin{array}{l}\text { Retrospective } \\
\text { cohort study }\end{array}$ & Urologic & 114 & 38 & 76 & $\begin{array}{l}\text { First } \\
\text { Nations, } \\
\text { Métis }\end{array}$ & Self-identified & $\begin{array}{l}\text { Morbidity after kidney } \\
\text { donation; death after } \\
\text { kidney donation }\end{array}$ \\
\hline Tonelli ${ }^{41}$ & $\begin{array}{l}\text { Retrospective } \\
\text { cohort study }\end{array}$ & Urologic & 9905 & 495 & 9410 & $\begin{array}{l}\text { First } \\
\text { Nations, } \\
\text { Inuit, or } \\
\text { Métis }\end{array}$ & $\begin{array}{l}\text { Canadian Organ } \\
\text { Replacement } \\
\text { Register }\end{array}$ & Rates of renal transplant \\
\hline Tonelli et al. ${ }^{42}$ & $\begin{array}{l}\text { Retrospective } \\
\text { cohort study }\end{array}$ & Urologic & 4840 & 685 & 4155 & $\begin{array}{l}\text { First } \\
\text { Nations, } \\
\text { Inuit, or } \\
\text { Métis }\end{array}$ & $\begin{array}{l}\text { Canadian Organ } \\
\text { Replacement } \\
\text { Register }\end{array}$ & Rates of renal transplant \\
\hline Weber et al. ${ }^{43}$ & $\begin{array}{l}\text { Retrospective } \\
\text { cohort study }\end{array}$ & Urologic & 705 & 126 & 579 & $\begin{array}{l}\text { First } \\
\text { Nation, } \\
\text { Inuit or } \\
\text { Métis }\end{array}$ & Self-identified & $\begin{array}{l}\text { Death after renal } \\
\text { transplant }\end{array}$ \\
\hline $\begin{array}{l}\text { Wei-Randall } \\
\text { et al. }{ }^{44}\end{array}$ & $\begin{array}{l}\text { Retrospective } \\
\text { cohort study }\end{array}$ & Cardiac & 353688 & 6560 & 347128 & $\begin{array}{l}\text { First } \\
\text { Nations }\end{array}$ & $\begin{array}{l}\text { Areas with high } \\
\text { percentage of } \\
\text { residents of First } \\
\text { Nations identity }\end{array}$ & $\begin{array}{l}\text { Rates of coronary } \\
\text { revascularization after } \\
\text { acute myocardial } \\
\text { infarction }\end{array}$ \\
\hline Yeates et al..$^{45}$ & $\begin{array}{l}\text { Retrospective } \\
\text { cohort study }\end{array}$ & Urologic & 24561 & 1071 & 23490 & $\begin{array}{l}\text { Not } \\
\text { Specified }\end{array}$ & $\begin{array}{l}\text { Canadian Organ } \\
\text { Replacement } \\
\text { Register }\end{array}$ & Rates of renal transplant \\
\hline Yeates et al. ${ }^{46}$ & $\begin{array}{l}\text { Retrospective } \\
\text { cohort study }\end{array}$ & Urologic & 17986 & 1650 & 16336 & $\begin{array}{l}\text { First } \\
\text { Nations, } \\
\text { Métis, Inuit }\end{array}$ & $\begin{array}{l}\text { Canadian Organ } \\
\text { Replacement } \\
\text { Register }\end{array}$ & Rates of renal transplant \\
\hline Zhang et al. ${ }^{47}$ & $\begin{array}{l}\text { Retrospective } \\
\text { cohort study }\end{array}$ & General & 149 & 20 & 129 & $\begin{array}{l}\text { First } \\
\text { Nations }\end{array}$ & $\begin{array}{l}\text { First Nations } \\
\text { status or self- } \\
\text { identification }\end{array}$ & $\begin{array}{l}\text { Death after liver } \\
\text { transplant }\end{array}$ \\
\hline
\end{tabular}

Twenty studies compared rates of surgery and wait times for Indigenous and non-Indigenous populations ${ }^{20-23,25,29-31,33-39,41,42,44-46}$ (Appendix 5, available at www.cmaj.ca/lookup/doi/10.1503/ cmaj.191682/tab-related-content). Six studies evaluating rates of renal transplantation found that Indigenous Peoples with endstage renal disease were less likely to receive a transplant (HR 0.34-0.54). ${ }^{25,34,41,42,45,46}$ Two studies found that Indigenous kidney transplant recipients experienced longer wait times by 3-7 months, ${ }^{43,46}$ and 4 of 5 studies evaluating rates of cesarean delivery found rates 3\%-5\% lower among First Nations women. ${ }^{20,29,33,35,38}$ In people with cardiovascular disease, 2 studies found lower rates of angiography (OR $0.73,95 \% \mathrm{Cl} 0.62-$ 0.87 ), but similar rates of coronary bypass procedures for Indigenous Peoples, although another study found lower rates of any cardiac surgery for Indigenous Peoples, including among those living in urban areas (0.31 v. 1.04 per 1000 people). 22,39,44 Three studies of amputation for diabetic complications found rates of amputation to be higher for First Nations and Métis Peoples. ${ }^{30,31,36}$ Among patients with osteoarthritis, Indigenous Peoples had half the rates of hip or knee arthroplasty than nonIndigenous patients. ${ }^{21}$

\section{Risk of bias}

Overall, 5 studies were considered to have low risk of bias, 9 studies were moderate, 2 studies were serious and 12 studies had an unclear risk of bias (Table 3). In most studies considered to have moderate or serious risk of bias, the method used to determine Indigenous identity introduced bias. Most studies attempted to adjust for potential confounders, such as area of residence, severity of disease and comorbidities, and as a result, had a low to moderate risk of bias within these categories.

\section{Interpretation}

In this systematic review of studies estimating the association between Indigenous identity and surgical rates or outcomes in Canada, we identified evidence of inequities for Indigenous Peoples. We identified few studies that directly addressed postoperative outcomes and our ability to draw conclusions on mortality and complications was limited by risk of bias, heterogeneity and the substantial underrepresentation of Inuit and Métis peoples. In the 4 studies (7 cohorts) suitable for meta-analysis, we identified an adjusted $30 \%$ higher risk of postoperative death 
Table 2 (part 1 of 2): Characteristics of studies that reported mortality outcomes

\begin{tabular}{|c|c|c|c|c|c|c|c|c|}
\hline Study & $\begin{array}{l}\text { Surgical } \\
\text { specialty }\end{array}$ & $\begin{array}{l}\text { Sample } \\
\text { size }\end{array}$ & $\begin{array}{l}\text { Mortality as } \\
\text { primary } \\
\text { outcome? }\end{array}$ & $\begin{array}{l}\text { Mortality } \\
\text { length of } \\
\text { follow-up }\end{array}$ & $\begin{array}{c}\text { Adjusted HR } \\
(95 \% \mathrm{Cl})^{\star}\end{array}$ & $\begin{array}{l}\text { Variables used in } \\
\text { adjustments }\end{array}$ & $\begin{array}{l}\text { Crude } \\
\text { mortality } \\
\text { rate }\end{array}$ & $\begin{array}{l}\text { Unadjusted } \\
\text { HR }(95 \% \mathrm{Cl})^{\star}\end{array}$ \\
\hline $\begin{array}{l}\text { Bresee et } \\
\text { al. }{ }^{22}\end{array}$ & Cardiac & 4287 & No & $\begin{array}{c}\text { Variable, } \\
\text { follow-up to } \\
\text { Mar. } 31,2009\end{array}$ & $\begin{array}{c}0.91 \\
(0.56-1.47)\end{array}$ & $\begin{array}{l}\text { Age, sex, diabetes, } \\
\text { hypertension, year of acute } \\
\text { MI, ejection fraction, smoking } \\
\text { status, coronary anatomy, } \\
\text { income quintiles, distance to } \\
\text { closest cardiac } \\
\text { catheterization laboratory, } \\
\text { comorbidities } \\
\text { (cerebrovascular disease, } \\
\text { heart failure, COPD, } \\
\text { dementia, HIV/AIDS, } \\
\text { metastatic cancer, mild liver } \\
\text { disease, moderate/severe } \\
\text { liver disease, paraplegia/ } \\
\text { hemiplegia, peptic ulcer } \\
\text { disease, peripheral vascular } \\
\text { disease, renal disease, } \\
\text { rheumatologic disease) }\end{array}$ & $\begin{array}{l}\text { Indigenous } \\
\text { 17/108; } \\
\text { non- } \\
\text { Indigenous } \\
997 / 4179\end{array}$ & $\begin{array}{c}0.67 \\
(0.42-1.07)\end{array}$ \\
\hline \multirow[t]{4}{*}{$\begin{array}{l}\text { Leslie et } \\
\text { al. }{ }^{28}\end{array}$} & $\begin{array}{l}\text { Orthopedics } \\
\text { (hip fracture) }\end{array}$ & 4145 & Yes & Unknown & $\begin{array}{c}1.37 \\
(1.16-1.62)\end{array}$ & $\begin{array}{l}\text { Age (within } 5 \mathrm{yr} \text { ), sex, } \\
\text { diabetes, area of residence, } \\
\text { ADGs (none, } 1-2,3-5,>5 \text { ) }\end{array}$ & NR & NR \\
\hline & $\begin{array}{l}\text { Orthopedics } \\
\text { (wrist fracture) }\end{array}$ & 8216 & Yes & Unknown & $\begin{array}{c}1.53 \\
(1.31-1.79)\end{array}$ & $\begin{array}{l}\text { Age (within } 5 \mathrm{yr} \text { ), sex, } \\
\text { diabetes, area of residence, } \\
\text { ADGs (none, } 1-2,3-5,>5 \text { ) }\end{array}$ & NR & NR \\
\hline & $\begin{array}{l}\text { Orthopedics } \\
\text { (vertebral } \\
\text { fracture) }\end{array}$ & 3431 & Yes & Unknown & $\begin{array}{c}1.30 \\
(1.01-1.67)\end{array}$ & $\begin{array}{l}\text { Age (within } 5 \mathrm{yr} \text { ), sex, } \\
\text { diabetes, area of residence, } \\
\text { ADGs (none, } 1-2,3-5,>5 \text { ) }\end{array}$ & NR & NR \\
\hline & $\begin{array}{l}\text { Orthopedics } \\
\text { (hip fracture) }\end{array}$ & 10367 & Yes & $12 \mathrm{mo}$ & $\begin{array}{l}\text { (OR) } 0.77 \\
(0.53-1.12)\end{array}$ & $\begin{array}{l}\text { Age (within } 5 \mathrm{yr} \text { ), sex, } \\
\text { diabetes, area of residence, } \\
\text { ADGs (none, } 1-2,3-5,>5 \text { ) }\end{array}$ & NR & NR \\
\hline $\begin{array}{l}\text { Sood et } \\
\text { al. }{ }^{39}\end{array}$ & $\begin{array}{l}\text { Cardiac } \\
\text { (mixed) }\end{array}$ & 12170 & Yes & In hospital & $\begin{array}{l}\text { (OR) } 1.15 \\
(0.63-2.08)\end{array}$ & $\begin{array}{l}\text { Age, sex, body mass index, } \\
\text { distance from centre, } \\
\text { comorbidities (smoking, } \\
\text { family history, diabetes, } \\
\text { lipids, chronic kidney } \\
\text { disease, dialysis, } \\
\text { hypertension, pulmonary } \\
\text { hypertension, } \\
\text { cerebrovascular accident, } \\
\text { COPD, peripheral vascular } \\
\text { disease, CHF, arrhythmia, } \\
\text { ACS, previous cardiac } \\
\text { procedure, functional status } \\
\text { (CCS class, NYHA class) } \\
\text { procedure type and urgency, } \\
\text { and medications } \\
\text { (angiotensin-converting } \\
\text { enzyme inhibitors, } \\
\beta \text {-blockers, ASA, steroids, } \\
\text { inotropic agents) }\end{array}$ & NR & $\begin{array}{c}\text { (OR) } 1.11 \\
(0.66-1.86)\end{array}$ \\
\hline $\begin{array}{l}\text { Zhang et } \\
\text { al. }^{47}\end{array}$ & $\begin{array}{l}\text { General } \\
\text { (Liver transplant) }\end{array}$ & 149 & Not stated & $22 \mathrm{yr}$ & NR & NA & $\begin{array}{c}\text { Indigenous } \\
6 / 20 ; \\
\text { non- } \\
\text { Indigenous } \\
26 / 129\end{array}$ & NR \\
\hline $\begin{array}{l}\text { Goulet et } \\
\text { al. }^{26}\end{array}$ & $\begin{array}{l}\text { Vascular } \\
\text { (revascularization } \\
\text { for peripheral } \\
\text { vascular disease) }\end{array}$ & 678 & Not stated & $5 \mathrm{yr}$ & $\begin{array}{c}1.00 \\
(0.6-1.6)\end{array}$ & Unknown & $\begin{array}{l}\text { Indigenous } \\
\text { 20/84; } \\
\text { non- } \\
\text { Indigenous } \\
\text { 160/594 }\end{array}$ & $\begin{array}{c}1.00 \\
(0.6-1.6)\end{array}$ \\
\hline
\end{tabular}


Table 2 (part 2 of 2): Characteristics of studies that reported mortality outcomes

\begin{tabular}{|c|c|c|c|c|c|c|c|c|}
\hline Study & $\begin{array}{l}\text { Surgical } \\
\text { specialty }\end{array}$ & $\begin{array}{l}\text { Sample } \\
\text { size }\end{array}$ & $\begin{array}{c}\text { Mortality as } \\
\text { primary } \\
\text { outcome? }\end{array}$ & $\begin{array}{l}\text { Mortality } \\
\text { length of } \\
\text { follow-up }\end{array}$ & $\begin{array}{c}\text { Adjusted HR } \\
(95 \% \mathrm{CI})^{\star}\end{array}$ & $\begin{array}{l}\text { Variables used in } \\
\text { adjustments }\end{array}$ & $\begin{array}{l}\text { Crude } \\
\text { mortality } \\
\text { rate }\end{array}$ & $\begin{array}{l}\text { Unadjusted } \\
\text { HR }(95 \% \mathrm{Cl})^{\star}\end{array}$ \\
\hline $\begin{array}{l}\text { Hong et } \\
\text { al. }{ }^{27}\end{array}$ & $\begin{array}{l}\text { General } \\
\text { (liver transplant) }\end{array}$ & 1164 & $\begin{array}{l}\text { Described as } \\
\text { "survival and } \\
\text { determinants } \\
\text { of survival" }\end{array}$ & $1 \mathrm{yr}$ & $\begin{array}{c}1.09 \\
(1.04-1.15)\end{array}$ & $\begin{array}{l}\text { Age, gender, ethnicity, ABO } \\
\text { blood group, donor type, } \\
\text { medical status before } \\
\text { transplantation, and hepatitis } \\
\text { B virus infection status }\end{array}$ & NR & NR \\
\hline $\begin{array}{l}\text { Weber et } \\
\text { al. }^{43}\end{array}$ & $\begin{array}{l}\text { Urology } \\
\text { (Kidney } \\
\text { transplant) }\end{array}$ & 705 & Not stated & $10 \mathrm{yr}$ & $\begin{array}{c}1.93 \\
(1.34-2.76)\end{array}$ & $\begin{array}{l}\text { Donor age }>45 \mathrm{yr}(\mathrm{v} .<45) \text {, } \\
\text { recipient age, recipient } \\
\text { gender, diabetes } \\
\text { pretransplant, deceased } \\
\text { donor (v. living donor), } \\
\text { delayed graft function, } \\
\text { immunosuppressive era, } \\
\text { maximum peak PRA, PTDM, } \\
\text { HLA disparity and } \\
\text { nonadherence post- } \\
\text { transplant. }\end{array}$ & NR & NR \\
\hline $\begin{array}{l}\text { Callegari et } \\
\text { al. }{ }^{23}\end{array}$ & $\begin{array}{l}\text { Plastics } \\
\text { (burn treatment) }\end{array}$ & 825 & Not stated & In hospital & NR & NA & $\begin{array}{l}\text { Indigenous } \\
4 / 80 \\
\text { non- } \\
\text { Indigenous } \\
26 / 745\end{array}$ & NR \\
\hline
\end{tabular}

Note: $\mathrm{ACS}=$ acute coronary syndrome, $\mathrm{ADG}=$ Aggregated Diagnosis Groups, ASA = acetylsalicylic acid, $\mathrm{CCS}=\mathrm{Canadian}$ Cardiovascular Society angina grading scale, $\mathrm{CHF}=$ congestive heart failure, $\mathrm{Cl}=$ confidence interval, $\mathrm{COPD}=$ chronic obstructive pulmonary disease, $\mathrm{HLA}=$ human leukocyte antigen, $\mathrm{HR}=$ hazard ratio, $\mathrm{MI}=\mathrm{myocardial}$ infarction, $\mathrm{NA}=$ not applicable, $\mathrm{NYHA}=$ New York Heart Association classification of heart failure, $\mathrm{NR}=$ not reported, $\mathrm{OR}=$ odds ratio, $\mathrm{PRA}=$ panel reactive antibody, PTDM $=$ posttransplantion diabetes mellitus.

*Unless indicated otherwise.

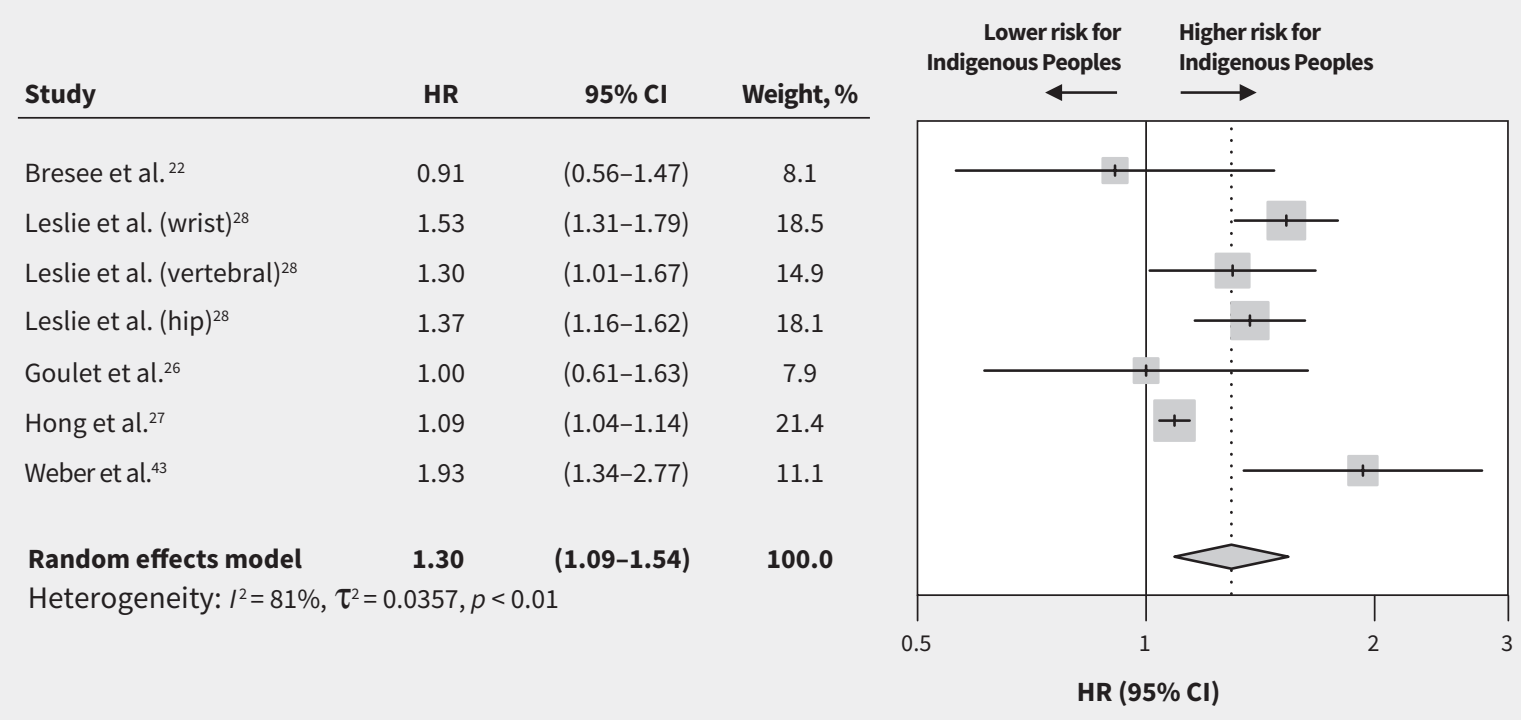

Figure 2: Forest plot of hazard ratios (HRs) and 95\% confidence intervals (Cls) of adjusted association of Indigenous identity with postoperative survival.

for Indigenous Peoples, as well as higher rates of complications, including postoperative infection and hospital readmission. Indigenous Peoples also appeared to have lower utilization rates of both elective surgeries aimed at improving quality of life, such as joint replacement, as well as potentially life-saving procedures, such as cardiac surgery, transplant and cesarean delivery.
Our findings are consistent with inequities in surgical outcomes for Indigenous Peoples observed in other high income countries. ${ }^{48-51}$ Although part of this effect may be attributable to the burden of chronic disease in Indigenous populations, our findings emerged by pooling results from studies that adjusted for important confounders, such as comorbidity status, which suggests that other factors may contribute. 
Table 3: Risk of bias

\begin{tabular}{|c|c|c|c|c|c|c|c|}
\hline Study & $\begin{array}{l}\text { Bias from } \\
\text { confounding }\end{array}$ & $\begin{array}{c}\text { Bias from } \\
\text { selection of } \\
\text { participants }\end{array}$ & $\begin{array}{c}\text { Bias from } \\
\text { measurement } \\
\text { of outcomes }\end{array}$ & $\begin{array}{c}\text { Bias from } \\
\text { measurement } \\
\text { of exposure }\end{array}$ & $\begin{array}{c}\text { Bias from } \\
\text { selection of } \\
\text { reported result }\end{array}$ & $\begin{array}{l}\text { Bias from } \\
\text { missing data }\end{array}$ & $\begin{array}{c}\text { Overall risk } \\
\text { of bias }\end{array}$ \\
\hline Aljohani et al. ${ }^{20}$ & Low & Low & Low & Moderate & Low & Unclear & Unclear \\
\hline Barnabe et al. ${ }^{21}$ & Moderate & Low & Low & Moderate & Low & Unclear & Unclear \\
\hline Bresee et al. ${ }^{22}$ & Low & Low & Low & Moderate & Low & Low & Moderate \\
\hline Callegari et al..$^{23}$ & Serious & Low & Low & Unclear & Low & Unclear & Unclear \\
\hline Cohen et al. ${ }^{24}$ & Moderate & Low & Low & Moderate & Low & Unclear & Unclear \\
\hline Dyck et al. ${ }^{25}$ & Serious & Low & Serious & Moderate & Low & Unclear & Unclear \\
\hline Goulet et al. ${ }^{26}$ & Moderate & Low & Low & Moderate & Low & Unclear & Unclear \\
\hline Hong et al. ${ }^{27}$ & Moderate & Low & Low & Low & Low & Low & Moderate \\
\hline Leslie et al. ${ }^{28}$ & Low & Low & Low & Moderate & Low & Low & Moderate \\
\hline Liu et al. ${ }^{29}$ & Low & Moderate & Low & Serious & Low & Unclear & Unclear \\
\hline Martens et al. ${ }^{30}$ & Low & Moderate & Low & Moderate & Low & Unclear & Unclear \\
\hline McIntyre et al. ${ }^{31}$ & Low & Moderate & Low & Low & Low & Low & Moderate \\
\hline Meatherall, ${ }^{32}$ & Serious & Unclear & Serious & Unclear & Low & Unclear & Unclear \\
\hline Oster et al..$^{33}$ & Moderate & Low & Low & Moderate & Low & Low & Moderate \\
\hline Promislow et al. ${ }^{34}$ & Low & Low & Low & Low & Low & Low & Low \\
\hline Riddell et al. ${ }^{35}$ & Low & Low & Low & Moderate & Low & Low & Moderate \\
\hline Rose et al. ${ }^{36}$ & Low & Moderate & Low & Unclear & Low & Unclear & Unclear \\
\hline Roy et al. ${ }^{37}$ & Serious & Moderate & Low & Unclear & Low & Unclear & Unclear \\
\hline Shen et al. ${ }^{38}$ & Moderate & Serious & Low & Moderate & Low & Low & Serious \\
\hline Sood et al. ${ }^{39}$ & Low & Low & Low & Low & Low & Low & Low \\
\hline Storsely et al. ${ }^{40}$ & Moderate & Low & Low & Low & Low & Low & Moderate \\
\hline Tonelli ${ }^{41}$ & Low & Low & Moderate & Low & Low & Moderate & Moderate \\
\hline Tonelli et al. ${ }^{42}$ & Moderate & Low & Low & Low & Low & Low & Moderate \\
\hline Weber et al. ${ }^{43}$ & Low & Low & Low & Low & Low & Low & Low \\
\hline Wei-Randall et al. ${ }^{44}$ & Serious & Low & Low & Serious & Low & Moderate & Serious \\
\hline Yeates $^{45}$ & Low & Low & Low & Low & Low & Low & Low \\
\hline Yeates $^{46}$ & Low & Low & Low & Low & Low & Low & Low \\
\hline Zhang et al. ${ }^{47}$ & Serious & Low & Low & Low & Low & Unclear & Unclear \\
\hline
\end{tabular}

Although access to surgical care is an essential element of a high-functioning health care system, the assessment of surgical need and provision of access to appropriate surgical services is complex. Currently, the literature provides limited data on wait times and rates of surgical procedures, which are proxy measures of access. The studies included in this review documented lower rates of common surgeries that can substantially affect health for both geographically isolated and urban Indigenous Peoples, but the results also raise questions about differences in disease stage at initial presentation and referral patterns. For example, access to intermediate types of care, including management of risk factors, could delay life-altering outcomes, such as limb amputations or the development of complications in living kidney donors.
It is essential to develop standardized national monitoring of surgical access and outcomes for Indigenous Peoples through culturally appropriate methods. Indigenous Peoples in Canada are diverse, and any pan-Indigenous research must consider the unique historic, geographic and cultural differences of First Nations, Inuit and Métis communities, as well as the heterogeneity within each of these distinct populations. The effective management of surgical diseases requires successful screening, diagnosis, timely access to surgical facilities and an appropriate transition to follow-up care. The inherent complexities in providing quality surgical care to a culturally diverse and geographically distributed population with higher rates of adverse outcomes highlights the urgent need for high quality, culturally aligned research across surgical specialties. Such 
research should assess access to and use of surgical care for Indigenous Peoples and the potential geographical, cultural, physical and systemic barriers to appropriate access.

The health of communities requires investment in the social determinants of health that, in addition to the availability of health services, include quality early childhood development, investment in culture and language, improved income distribution, quality housing and access to personal safety and security, education, food security, mental wellness and a healthy environment. To address the differences in outcomes identified in this study, there is a need to recognize the colonial conditions in which the Canadian health care system was established and continues to operate. The current system of health care delivery in Canada creates systemic barriers to care and limits the ability to describe national patterns in Indigenous health outcomes. ${ }^{52} \mathrm{~A}$ comprehensive national plan must be developed to improve access to surgical services and to measure, monitor and improve surgical, obstetric and anesthesia outcomes for all Canadians, with a focus on Indigenous Peoples and those that live in rural and remote locations. ${ }^{53}$ Indigenous leaders and community voices should be at the centre of this discussion.

\section{Limitations}

Some studies included in this review date back to 1989, when postoperative care may have been different from today. The oldest study included in the meta-analysis was published in 2006 , however. No studies specifically addressed postoperative outcomes in patients who identified as Inuit or Métis. Most studies were retrospective and many were biased. The methods for determining Indigenous identity were heterogeneous, with the potential for misclassification. This denominator bias likely underestimates the disparities shown in this study. ${ }^{54}$ The severity or stage of surgical disease was not known at presentation. We recognize, for example, that death attributed to wrist surgery is unusual, and we believe this to reflect unmeasured confounding and the lower baseline health status of Indigenous patients. Unfortunately, many studies did not report unadjusted mortality rates or effect measures including the majority of the studies included in our primary adjusted meta-analysis. Therefore, there were few data and little overlap among studies to support an unadjusted metaanalysis. A pooled, unadjusted effect measure for mortality or secondary outcomes was not provided, given the incomplete reporting in included studies, which precluded estimation of the impact of confounder adjustment on estimated effect sizes and limited part of our analysis to a narrative synthesis. Our metaanalysis pooled studies with variable windows of outcome ascertainment, although previous research suggests consistency in postoperative effect measures over time..$^{55}$ We also identified heterogeneity in our pooled estimate that appears to be attributable to data from studies of transplantation surgery. In studies of nontransplant surgery, heterogeneity was low and the pooled nontransplant effect size was similar to the overall pooled effect. We recognize that utilization rates are not a direct measure of access to surgical care as they fail to fully describe the various barriers faced by patients and do not account for personal choices made by Indigenous patients in response to their well-known lack of trust and cultural safety in the health care system.

\section{Conclusion}

In a systematic review of surgical care utilization and outcomes, we found that Canadian Indigenous Peoples had higher rates of mortality and adverse events, with lower rates of surgical utilization. First Nations, Inuit and Métis communities are increasingly prepared to direct Indigenous population health research and policy. The rights and benefits of research to Indigenous communities, colonial policies and anti-Indigenous racism should be considered in any analysis of health policy and outcomes research.

\section{References}

1. Global surgery 2030. Policy brief: assessing access, indicators for a healthy surgical system. Lancet Commission on Global Surgery; 2015.

2. Shrime MG, Bickler SW, Alkire BC, et al. Global burden of surgical disease: an estimation from the provider perspective. Lancet Glob Health 2015;3(Suppl 2):S8-9.

3. Aboriginal peoples in Canada: key results from the 2016 census. Ottawa: Statistics Canada; 2017. Available: https://www150.statcan.gc.ca/n1/daily-quotidien /171025/dq171025a-eng.htm?indid=14430-1\&indgeo=0 (accessed 2021 Apr. 6).

4. Walker J, Lovett R, Kukutai T, et al. Indigenous health data and the path to healing. Lancet 2017;390:2022-3.

5. Katz A, Kinew KA, Star L, et al. The health status of and access to healthcare by registered First Nation Peoples in Manitoba. Winnipeg: Manitoba Centre for Health Policy; 2019.

6. Anderson I, Robson B, Connolly M, et al. Indigenous and tribal peoples' health (The Lancet-Lowitja Institute Global Collaboration): a population study. Lancet 2016;388:131-57.

7. First Nations regional longitudinal health survey. Ottawa: First Nations Centre; 2005.

8. King M, Smith A, Gracey M. Indigenous health part 2: the underlying causes of the health gap. Lancet 2009;374:76-85.

9. MacMillan HL, MacMillan AB, Offord DR, et al. Aboiginal health. CMAJ 1996; 155:1569-78.

10. Nelson SE, Wilson K. The mental health of Indigenous peoples in Canada: a critical review of research. Soc Sci Med 2017;176:93-112.

11. Young TK, Reading J, Elias B, et al. Type 2 diabetes mellitus in Canada's first nations: status of an epidemic in progress. [published erratum in CMAJ 2000; 163:1132]. CMAJ 2000;163:561-6.

12. Patterson M, Flinn S, Barker K. Addressing tuberculosis among Inuit in Canada. Canada Commun Dis Rep 2018;44:82-5.

13. Crowshoe L, Dannenbaum D, Green M, et al.; Diabetes Canada Clinical Practice Guidelines Expert Committee. Type 2 tiabetes and Indigenous Peoples. Can J Diabetes 2018;42:296-306.

14. Health Inequalities Data Tool. Ottawa: Public Health Agency of Canada, the Pan-Canadian Public Health Network, Statistics Canada and the Canadian Institute of Health Information; 2017. Available: https://health-infobase.can ada.ca/health-inequalities/data-tool/index (accessed 2019 Nov. 22).

15. Subedi R, Greenberg TL, Roshanafshar S. Does geography matter in mortality? An analysis of potentially avoidable mortality by remoteness index in Canada. Health Rep 2019;30:3-15.

16. Stroup DF, Berlin JA, Morton SC, et al. Meta-analysis of observational studies in epidemiology: a proposal for reporting. JAMA 2000;283:2008-12.

17. Moher D, Liberati A, Tetzlaff J, et al.; The PRISMA Group. Preferred reporting items for systematic reviews and meta-analyses: the PRISMA statement. PLOS Med 2009;6: e1000097.

18. McGowan J, Sampson M, Salzwedel DM, et al. PRESS peer review of electronic search strategies: 2015 guideline statement. J Clin Epidemiol 2016;75:40-6.

19. Higgins JPT, Altman DG, Gøtzsche PC, et al. The Cochrane Collaboration's tool for assessing risk of bias in randomised trials. BMJ 2011;343:d5928.

20. Aljohani N, Rempel BM, Ludwig S, et al. Impact of diabetes on maternal-fetal outcomes in Manitoba: Relationship with ethnic and environmental factors. Clin Invest Med 2008;31:E338-E45.

21. Barnabe C, Hemmelgarn B, Jones CA, et al. Imbalance of prevalence and specialty care for osteoarthritis for First Nations people in Alberta, Canada. J Rheumatol 2015;42:323-8.

22. Bresee LC, Knudtson ML, Zhang J, et al. Likelihood of coronary angiography among First Nations patients with acute myocardial infarction. CMAJ 2014;186:E372-80.

23. Callegari PR, Alton JDM, Shankowsky HA, et al. Burn injuries in native Canadians: a 10-year experience. Burns Incl Therm Inj 1989;15:15-9. 
24. Cohen MM, Young TK, Hammarstrand KM. Ethnic variation in cholecystectomy rates and outcomes, Manitoba, Canada, 1972-84. Am J Public Health 1989;79:751-5.

25. Dyck RF, Tan L. Rates and outcomes of diabetic end-stage renal disease among registered native people in Saskatchewan. CMAJ 1994;150:203-8.

26. Goulet S, Trepman E, Cheang M, et al. Revascularization for peripheral vascular disease in Aboriginal and non-Aboriginal patients. J Vasc Surg 2006;43:735-41.

27. Hong Z, Wu J, Smart G, et al. Survival analysis of liver transplant patients in Canada 1997-2002. Transplant Proc 2006;38:2951-6.

28. Leslie WD, Brennan SL, Prior HJ, et al. The contributions of First Nations ethnicity, income, and delays in surgery on mortality post-fracture: a populationbased analysis. Osteoporos Int 2013;24:1247-56.

29. Liu SL, Shah BR, Naqshbandi M, et al. Increased rates of adverse outcomes for gestational diabetes and pre-pregnancy diabetes in on-reserve First Nations women in Ontario, Canada. Diabet Med 2012;29:e180-3.

30. Martens PJ, Martin BD, O'Neil JD, et al. Diabetes and adverse outcomes in a first nations population: associations with healthcare access, and socioeconomic and geographical factors. Can J Diabetes 2007;31:223-32.

31. McIntyre I, Boughen C, Trepman E, et al. Foot and ankle problems of Aboriginal and non-Aboriginal diabetic patients with end-stage renal disease. Foot Ankle Int 2007;28:674-86.

32. Meatherall BL, Garrett MR, Kaufert J, et al. Disability and quality of life in Canadian Aboriginal and non-Aboriginal diabetic lower-extremity amputees. Arch Phys Med Rehabil 2005;86:1594-602.

33. Oster RT, Toth EL. Longitudinal rates and risk factors for adverse birth weight among First Nations pregnancies in Alberta. J Obstet Gynaecol Can 2016;38:29-34.

34. Promislow $S$, Hemmelgarn B, Rigatto $C$, et al. Young Aboriginals are less likely to receive a renal transplant: a Canadian national study. BMC Nephrol 2013;14:11.

35. Riddell CA, Hutcheon JA, Dahlgren LS. Differences in obstetric care among nulliparous First Nations and non-First Nations women in British Columbia, Canada. CMAJ 2016;188:E36-43.

36. Rose G, Duerksen F, Trepman E, et al. Multidisciplinary treatment of diabetic foot ulcers in Canadian Aboriginal and non-Aboriginal people. Foot Ankle Surg 2008;14:74-81.

37. Roy M. Analysis of uveitis in a Canadian Aboriginal population. Can J Ophthalmol 2014;49:128-34.

38. Shen GX, Shafer LA, Martens PJ, et al. Does First Nations ancestry modify the association between gestational diabetes and subsequent diabetes: a historical prospective cohort study among women in Manitoba, Canada. Diabet Med 2016;33:1245-52.

39. Sood MM, Tangri N, Komenda P, et al. Incidence, secular trends, and outcomes of cardiac surgery in Aboriginal peoples. Can J Cardiol 2013;29:1629-36.
40. Storsley LJ, Young A, Rush DN, et al. Long-term medical outcomes among Aboriginal living kidney donors. Transplantation 2010;90:401-6.

41. Tonelli M, Hemmelgarn B, Kim AKJ, et al. Association between residence location and likelihood of kidney transplantation in Aboriginal patients treated with dialysis in Canada. Kidney Int 2006;70:924-30.

42. Tonelli M, Hemmelgarn B, Manns B, et al. Death and renal transplantation among Aboriginal people undergoing dialysis. CMAJ 2004;171:577-82.

43. Weber CLC, Rush DN, Jeffery JR, et al. Kidney transplantation outcomes in Canadian Aboriginals. Am J Transplant 2006;6:1875-81.

44. Wei-Randall HK, Josée Davidson M, Jin J, et al. Acute myocardial infarction hospitalization and treatment: areas with a high percentage of first nations identity residents. Health Rep 2013;24:3-10.

45. Yeates KE, Schaubel DE, Cass A, et al. Access to renal transplantation for minority patients with ESRD in Canada. Am J Kidney Dis 2004;44:1083-9.

46. Yeates KE, Cass A, Sequist TD, et al. Indigenous people in Australia, Canada, New Zealand and the United States are less likely to receive renal transplantation. Kidney Int 2009;76:659-64.

47. Zhang M, Uhanova J, Minuk GY. Liver transplant outcomes in a Canadian First Nations population. Can J Gastroenterol 2011;25:307-10.

48. Rahiri JL, Alexander Z, Harwood M, et al. Systematic review of disparities in surgical care for Maori in New Zealand. ANZ J Surg 2018;88:683-9.

49. Lawton PD, McDonald SP, Snelling PL, et al. Organ transplantation in Australia: inequities in access and outcome for Indigenous Australians. Transplantation 2017;101:e345-6.

50. Keddis MT, Sharma A, Ilyas M, et al. Transplant center assessment of the inequity in the kidney transplant process and outcomes for the indigenous American patients. PLoS One 2018;13:e0207819.

51. McLeod M, Signal V, Gurney J, et al. Postoperative mortality of Indigenous populations compared with non-Indigenous populations: a systematic review. JAMA Surg 2020;155:636-56.

52. Kirby M, LeBreton M. The health of Canadians - the federal role. Final report of the Standing Senate Committee on Social Affairs, Science and Technology. Ottawa; 2002.

53. Orser BA, Wilson CR. Canada needs a national strategy for anesthesia services in rural and remote regions. CMAJ 2020;192:E861-3.

54. Sarfati D, Garvey G, Robson B, et al. Measuring cancer in indigenous popula tions. Ann Epidemiol 2018;28:335-42.

55. Smith T, Li X, Nylander W, et al. Thirty-day postoperative mortality risk estimates and 1-year survival in Veterans Health Administration surgery patients. JAMA Surg 2016;151:417-22.
Competing interests: Dylan Bould is chair of the board of trustees of the Canadian Anesthesiologist's Society International Education Foundation. No other competing interests were declared.

This article has been peer reviewed.

Affiliations: Departments of Anesthesiology and Pain Medicine (McVicar, Poon, Bould, Mclsaac) and of Surgery (Kimmaliardjuk), Faculty of Medicine (Ahmad, during the conduct of the study); Centre for Health Law, Policy and Ethics (Nickerson), University of Ottawa; The Ottawa Hospital (McVicar, Poon, Kimmaliardjuk, Mclsaac); Children's Hospital of Eastern Ontario (Bould); Bruyère Research Institute (Nickerson), Ottawa, Ont.; Department of Surgery and Northern Medical Program (Caron), University of British Columbia, Prince George, BC; Centre for Excellence in Indigenous Health (Caron), University of British Columbia, Vancouver, BC; Department of Anesthesiology and Pain Medicine (Ahmad, at time of writing), University of Toronto, Toronto, Ont.; Qikiqtani General Hospital (Sheffield), Iqaluit, NU; West Parry Sound Health Centre (Champion), Parry Sound, Ont.; Department of Surgery (Champion), Northern Ontario School of Medicine, Sudbury, Ont.

Contributors: Nadine Caron, Dylan Bould, Jason Nickerson, Dan Mclsaac and Jason McVicar conceived of and designed the study. Alana Poon, Nora Ahmad, Jason McVicar and Dan Mclsaac interpreted the data and drafted the article. All of the authors revised the draft critically for important intellectual content and approved the final version.
Content licence: This is an Open Access article distributed in accordance with the terms of the Creative Commons Attribution (CC BY-NC ND 4.0) licence, which permits use, distribution and reproduction in any medium, provided that the original publication is properly cited, the use is noncommercial (i.e., research or educational use), and no modifications or adaptations are made. See: https://creativecommons. org/licenses/by-nc-nd/4.0/

Funding: This project was funded by a grant from The Ottawa Hospital Academic Medical Organization Innovation Fund, The Canadian Anesthesia Research Foundation. Daniel Mclsaac and Jason McVicar receive salary support from The Ottawa Hospital Department of Anesthesiology and Pain Medicine.

Data sharing: All study data are available through contact with the corresponding author.

Acknowledgements: The authors thank Sascha Davis for assistance with the search strategy as well as Jean Allen and Michelle DoucetteIssaluk for their expertise in reviewing the manuscript.

Disclaimer: Three of the authors identify as Indigenous: Jason McVicar (Métis), Nadine Caron (First Nations) and Donna May Kimmiliardjuk (Inuk).

Accepted: Feb. 1, 2021

Correspondence to: Jason McVicar, jmcvicar@toh.ca 